\title{
Defensoría del Pueblo, supervisión penitenciaria y reformas durante la COVID-19
}

Gustavo Raúl Ytokazu Minami

Abogado por la Universidad de Lima.

Magíster en Derecho por la Universidad de Kyushu. Doctorando en Desarrollo Internacional por la Universidad de Nagoya. Miembro Asociado de ADV Editores - Revista ADVOCATUS.

SUMARI0:

I. Introducción.

II. La capacidad del Estado, el Sistema Penitenciario peruano como Sistema Adaptativo Complejo.

III. Cárceles peruanas en crisis y la supervisión penitenciaria.

IV. Supervisión y Reformas Penitenciarias durante la pandemia.

1. Capacidad normativa.

2. Capacidad analítica.

3. Capacidad de entrega de resultados.

4. Capacidad de coordinación.

v. Conclusiones. 


\title{
RESUMEN:
}

El Sistema Penitenciario y sus reformas han experimentado dificultades en su formulación e implementación para cumplir con el mandato constitucional de reeducar, rehabilitar y reincorporar a los penados a la sociedad. Para ello, analizamos los desafíos de la capacidad administrativa del Estado respecto al Sistema Penitenciario Peruano en su condición de sistema adaptativo complejo y la supervisión penitenciaria a cargo de la Defensoría del Pueblo durante la pandemia. Concluimos que una reforma integral debe incluir a la Defensoría del Pueblo como Mecanismo Nacional de Prevención de la Tortura (MNP) y que ésta a su vez congregue a otros actores del Estado y la sociedad civil para la ejecución de acciones sostenibles a largo plazo.

Palabras clave: prisión, reformas, Covid-19, Teoría de la Complejidad, sistemas adaptativos complejos, capacidad administrativa, sistema penitenciario, Perú, Mecanismo Nacional de Prevención de la Tortura, Defensoría del Pueblo, INPE

\begin{abstract}
:
The penitentiary system and its reforms have struggled with their formulation and implementation in order to fulfill its constitutional mandate of re-education, rehabilitation and reincorporation of former convicts into society. We present an analysis of the challenges that the State's administrative capacity faces towards the Peruvian Penitentiary System in its complex adaptive system and the penitentiary supervision during the pandemic under charge of the 0 mbudsman. We conclude that an integral reform includes the Ombudsman as a National Torture Prevention Mechanism (NPM), and as a congregator for other State and Civil Society Actors for the implementation of long-term sustainable actions.

Keywords: prison, reforms, Covid-19, Complexity Theory, Complex adaptive systems, penitentiary system, Peru, National Torture Prevention Mechanism, 0mbudsman, INPE
\end{abstract}

"One of the tests of the civilization of people is the treatment of its criminals".

"Una de las pruebas de la civilización de las personas es el trato a sus criminales".

\section{INTRODUCCIÓN}

Durante el Gobierno de Ollanta Humala se emitieron 10 Medidas de Reforma del Sistema Penitenciario enfocadas a solucionar el hacinamiento carcelario y la corrupción en abril $2012^{2}$ a pesar de la existencia de un marco normativo vigente. Los siguientes gobiernos promulgaron normas, políticas y planes prospectivos adicio- nales, incluyendo la reiterada declaración de emergencia del Sistema Penitenciario, ${ }^{3}$ siendo la última vigente hasta el 6 de enero de $2021^{4}$. Sin embargo, la declaración del estado de las cosas inconstitucional en materia de salud mental penitenciaria y hacinamiento de establecimientos penitenciarios evidencia que aún existe mucho por mejorar. ${ }^{5}$ En virtud a ello, el Tribunal Constitucional exhortó al Ministerio de Justicia y Derechos Humanos - MINJUS, a elaborar un nuevo Plan Nacional de Política Penitenciaria, establecer mecanismos para erradicar el hacinamiento, desarrollar estrategias para superar las deficiencias de infraestructura y reestructurar el Instituto

1. Rutherford Birchard Hayes, Diary and Letters of Rutherford Birchard Hayes, vol. V, cap. LIV, ed. Charles Richard WiIliams (Columbus, Ohio State Archeological and Historical Society, 1926).

2. Mediante nota de prensa $N^{\circ}$ 34-2012-INPE con fecha 13 de abril de 2012, el MINJUS e INPE dieron a conocer las 10 Medidas de Reforma del Sistema Penitenciario.

Las 10 Medidas de Reforma del Sistema Penitenciario consideraban (i) la lucha contra la corrupción, (ii) reducción del hacinamiento, (iii) seguridad penitenciaria, (iv) salud en los centros penitenciarios, (v) mejora del tratamiento penitenciario, (vi) fortalecimiento de la gestión del INPE, (vii) mejora del personal del INPE, (viii) participación del sector privado en materia penitenciaria, (ix) acciones de prevención y reducción del delito, y (x) la implementación del Programa CREO como eje de un nuevo modelo penitenciario.

Ministerio de Justicia y Derechos Humanos e Instituto Nacional Penitenciario, "10 Medidas de Reforma del Sistema Penitenciario", 13 de abril de 2012, http://www2.congreso.gob.pe/sicr/cendocbib/con4_uibd.nsf/810635CDF2139A7F052581300073F277/\$FILE/10_medidas.pdf

3. Declaran en emergencia al Instituto Nacional Penitenciario, por razones de seguridad e interés público, Decreto de Urgencia 04-2005. 
Nacional Penitenciario - INPE, para mejorar la reeducación, rehabilitación y reinserción del penado a más tardar en el año 2025. Caso contrario, los establecimientos penitenciarios hacinados deberán ser cerrados bajo responsabilidad de la omisión o deficiencia de las autoridades competentes, empezando por el MINJUS. Por ello, el MINJUS aprobó el Plan Nacional de Política Penitenciaria al $2030^{6}$, quedando pendientes las otras obligaciones requeridas por el Tribunal Constitucional.

En este contexto, la Defensoría del Pueblo es el organismo constitucional autónomo a cargo de la supervisión doméstica penitenciaria. Para ello, la Constitución del Perú otorga a la Defensoría del Pueblo el mandato para defender los derechos de los ciudadanos y denunciar aquellos asuntos relacionados con la afectación de derechos fundamentales desde su creación. A partir de la implementación de las normas del Protocolo Facultativo de la Convención contra la Tortura - $\mathrm{PFCCT},{ }^{7}$ mediante la designación de la Defensoría del Pueblo como Mecanismo Nacional de Prevención de la Tortura - MNP, sus funciones han sido fortalecidas a nivel nacional para la supervisión de la población vulnerable privada de la libertad. ${ }^{8}$ Sin embargo, dicha entidad solo emite recomendaciones de carácter no vinculantes y para ello resulta necesario explorar su accionar en el Sistema Penitenciario, particularmente durante la pandemia.

Los Defensores del Pueblo poseen el rol como reserva moral siendo imparciales respecto de los poderes del Estado, buscan que sus resoluciones sean tomadas en consideración. ${ }^{9}$ A pesar de que puede ser percibido como una limitación, el carácter no vinculante de sus recomendaciones permite hacer la diferencia en política para proteger intereses de poblaciones vulnerables o en entornos desfavorables dado que no necesariamente representan una amenaza para los congresistas o gobernantes y es consistente con los conceptos de legitimidad democrática. ${ }^{10}$

Declaran en emergencia el Sistema Penitenciario y dictan medidas excepcionales y urgentes en materia económica y financiera, Decreto de Urgencia 007-2012.

Decreto Legislativo que declara en emergencia y dicta medidas para la reestructuración del Sistema Nacional Penitenciario y el Instituto Nacional Penitenciario, Decreto Legislativo 1325.

4. Decreto Supremo que prorroga la emergencia dispuesta por el Decreto Legislativo 1325, para la reestructuración del Sistema Nacional Penitenciario y el Instituto Nacional Penitenciario, Decreto Supremo Nº 013-2018-JUS.

5. La sentencia del Tribunal Constitucional relativa a (i) M.H.F.C. representada por Melchora Castañeda Tuesta de Flores c/ Director del INPE publicada en la página web del Tribunal Constitucional el 16 de septiembre de 2019; y (ii) Pedro Gonzalo Marroquín Soto representado por Miguel Aurelio Baca Villar c/ Director del INPE (2010), publicada en el Diario Oficial El Peruano el 3 de noviembre de 2010 se pronuncia sobre el estado de cosas inconstitucional en materia de prestación de servicios de salud mental en establecimientos penitenciarios.

La sentencia del Tribunal Constitucional sobre el caso C.C.B. contra el Establecimiento Penitenciario de Tacna (2020) publicada en el Diario Oficial El Peruano el 4 de junio de 2020 se pronuncia sobre el estado de cosas inconstitucional por el hacinamiento penitenciario.

6. Decreto Supremo que aprueba la Política Nacional Penitenciaria al 2030, Decreto Supremo º 011-2020-JUS.

7. La República del Perú es signataria de la Convención de las Naciones Unidas contra la Tortura, vigente en el país desde el 6 de agosto de 1988 y de su Protocolo Facultativo de la Convención contra la Tortura vigente desde el 14 de octubre de 2006.

8. La Ley que amplía las funciones del Defensor del Pueblo como órgano encargado del Mecanismo Nacional de Prevención contra la Tortura y otros Tratos o Penas Crueles, Inhumanos o Degradantes, Ley 30394, amplió las funciones del Defensor del Pueblo como MNP.

9. Fredrik Uggla, "The Ombudsman in Latin America", Journal of Latin American Studies 36, no. 3, (2004): 427-428.

10. Ludvig Beckman y Frederik Uggla, "An Ombudsman for Future Generations: Legitimate and Effective?" En: Institutions for Future Generations, eds. Iñigo González-Ricoy y Axel Gosseries (Oxford: Oxford University Press, 2017$)$ : 131. 
Figura 1.1. Confianza en las instituciones peruanas: Julio-Diciembre 2018 / Julio-Diciembre 2019

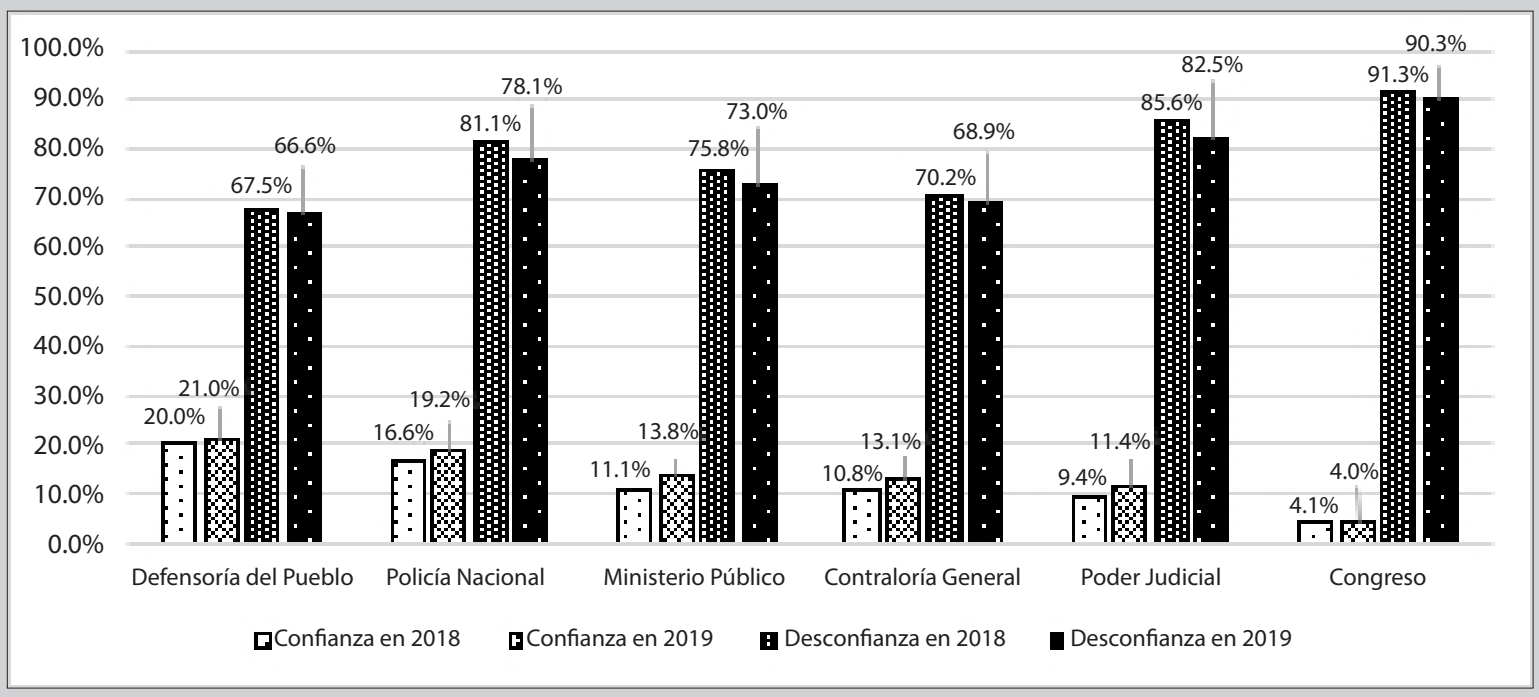

Fuente: Información del INEI"11

La percepción de la ciudadanía peruana sobre las instituciones públicas presentada en la Figura 1.1 muestra una desconfianza generalizada en las instituciones involucradas en la administración de justicia, ejecución penal, dación de políticas públicas y leyes, y la rendición de cuentas. No obstante ello, la Defensoría del Pueblo obtuvo una mejor percepción de confianza en 2018 y 2019 en comparación con la Policía Nacional, Ministerio Público, Poder Judicial Contraloría y el Congreso. Sin perjuicio de ello, la corrupción aún se mantiene como una tarea pendiente en la Administración Pública. Según la Figura 1.2., si bien la Defensoría del Pueblo es percibida como una de las instituciones con mayor enfoque contra la corrupción, la percepción "muy buena/buena" disminuyó del 2012 al 2019 en un 25\%. Así las cosas, el Poder Judicial y el Congreso poseen una alta desconfianza por parte de la población peruana, y ambas instituciones son percibidas con un bajo rendimiento en sus esfuerzos por reducir la corrupción.

\section{Figura 1.2. Percepción "Muy Buena/Buena" de la Respuesta Institucional contra la Corrupción}

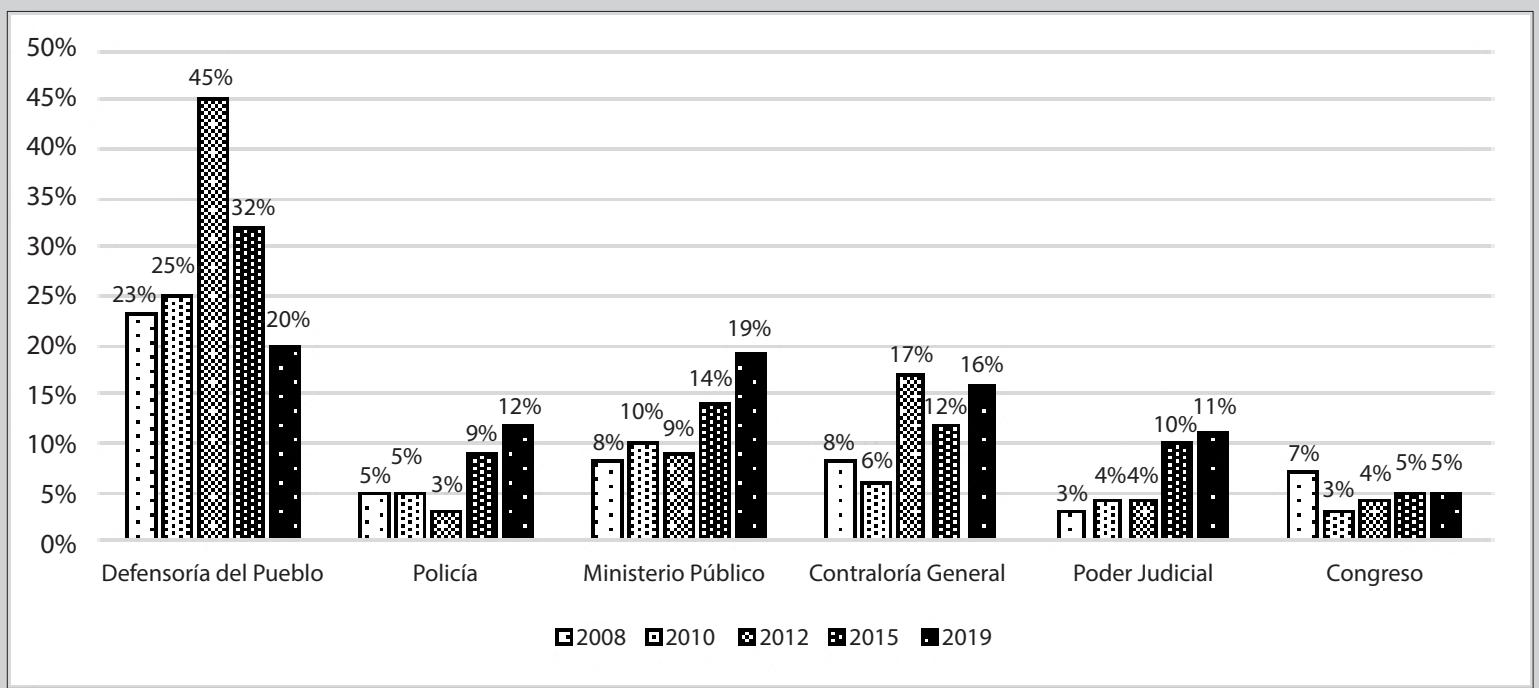

Fuente: Información de Proética ${ }^{12}$ 
El 6 de marzo de 2020 fue anunciado el primer paciente diagnosticado con la COVID-19 en el Perú y el Gobierno implementó medidas de emergencia en diferentes sectores del Estado. Si bien existen limitaciones en la información sobre los niveles de confianza hacia las instituciones y percepciones de corrupción durante la pandemia, ${ }_{13}^{13}$ consideramos necesario analizar al Sistema Penitenciario peruano en su condición de sistema adaptativo complejo conforme con la Teoría de la Complejidad, la supervisión penitenciaria a cargo de la Defensoría del Pueblo y las reformas del dicho sistema durante la primera etapa de la pandemia considerada como la primera ola. Por tal motivo la Sección II explora la capacidad del Estado peruano y el Sistema Penitenciario como un sistema adaptativo complejo; la Sección III examina la supervisión y reformas del Sistema Penitenciario peruano durante la COVID-19 y la Sección V detalla las conclusiones.

\section{LA CAPACIDAD DEL ESTADO Y EL SISTE- MA PENITENCIARIO PERUANO COMO SIS- TEMA ADAPTATIVO COMPLEJO}

La autoridad y el poder del Estado, la promulgación de políticas públicas y la dación de leyes están relacionados con el poder de infraestructura -infrastructural power-, que también está asociado con la capacidad del Estado. ${ }^{14}$ En el caso del Perú, el crecimiento económico y reducción de pobreza han permitido una mejora significativa en la capacidad del estado, desarrollada conjuntamente con el crecimiento de la informalidad, actividades ilícitas y delictivas. ${ }^{15}$

Bajo dicho contexto, consideramos analizar la capacidad administrativa para dar soluciones dentro del Estado, tales como la prestación de servicios públicos - capacidad de prestación-, el estado de derecho y la aplicación de la ley - capacidad normativa-, la colaboración inte-

11. INEI, Perú: Percepción Ciudadana sobre Gobernabilidad, Democracia y Confianza en las Instituciones, Semestre Julio-Diciembre 2019, Febrero 2020, http://m.inei.gob.pe/media/MenuRecursivo/boletines/boletin_gobernabilidad_febrero2020.pdf ; INEI, Perú: Percepción Ciudadana sobre Gobernabilidad, Democracia y Confianza en las Instituciones, Semestre Julio-Diciembre 2018, Febrero 2019, http://m.inei.gob.pe/media/MenuRecursivo/boletines/boletin-percepcion-gobernabilidad-enero-2019-4ta-version.pdf

12. Proética: VI Encuesta Nacional sobre Percepciones de la corrupción en el Perú 2012 (2012), producido por Ipsos Opinión y Mercado S.A., https://www.proetica.org.pe/wp-content/uploads/2018/04/317736944-Septima-Encuesta-Nacional-sobre-percepciones-de-la-corrupcion-2012.pdf ; IX Encuesta Nacional sobre Percepciones de la corrupción en el Perú 2015 (2015), producido por Ipsos Opinión y Mercado S.A., https://www.proetica.org. pe/wp-content/uploads/2018/04/292794637-Novena-Encuesta-nacional-sobre-percepciones-de-la-corrupcion-2015.pdf ; XI Encuesta Nacional sobre Percepciones de la corrupción en el Perú 2019 (Diciembre 2019), producido por Instituto de Estudios Peruanos, https://www.proetica.org.pe/contenido/xi-encuesta-nacional-sobre-percepciones-de-la-corrupcion-en-el-peru-2019/

13. El último informe técnico del INEI sobre las percepción ciudadana y confianza hacia las instituciones comprende el periodo entre octubre 2019 y marzo 2020 y durante el 2020 no se llevó a cabo encuesta alguna sobre percepciones de la corrupción.

14. Bastián González-Bustamante, "Evolution and early government responses to COVID-19 in South America", World Development 137 (2021), 2.

15. Eduardo Dargent, Andreas E. Feldmann y Juan Pablo Luna, "Greater State Capacity, Lesser Stateness: Lessons from the Peruvian Commodity Boom", Politics \& Society 45 (2017): 10-11, 26 ; Juan Pablo Luna, "Estado en América Latina: Problemática y Agenda de Investigación," en Nuevos Enfoques para el Estudio de los Estados Latinoamericanos, ed. Pablo Andrade A. (Quito: Corporación Editora Nacional, 2020), 114. 
gral entre instituciones y autoridades estatales - capacidad de coordinación-, y la capacidad analítica para evaluar las previsiones y acciones estatales en curso $^{16}$ relacionadas con los asuntos penitenciarios bajo la Teoría de la Complejidad.

La Teoría de la Complejidad, por otro lado, ha sido desarrollada en las ciencias naturales, y puede ser implementada en la investigación socio-jurídica, tal y como lo propone la academia ${ }^{17}$ para el estudio de los sistemas dinámicos no lineales en virtud de los cuales las salidas - output- no son necesariamente proporcionales a las entradas —input- ${ }^{18}$. Así las cosas, esta teoría explora la complejidad entre las interacciones del conjunto de reglas, el sistema y su entorno.

El Sistema Penitenciario Peruano es un sistema adaptativo complejo representado por las propiedades generales propuestas por Ruhl' ${ }^{19}$ basadas en el trabajo de Holland ${ }^{20}$ tales como la agregación - aggregation-, no linealidad —nonlinearity -, flujos — flow-, diversidad — diversity- y la autocrítica — self-criticality-.

En primer lugar, la "agregación" se refiere a que los sistemas actúan en base a comportamientos complejos a gran escala que surgen de las interacciones agregadas de estos agentes menos complejos ${ }^{21} \mathrm{y}$ el Sistema Penitenciario ha surgido debido a su estructura actual de sistema auto organizado y a las interacciones entre el MINJUS, INPE, los trabajadores penitenciarios y los internos.

En segundo lugar, la "no linealidad" considera que las relaciones de los componentes del sistema no exhiben una proporcionalidad matemática ${ }^{22}$, lo cual es observado en la emergencia permanente del sistema penitenciario para implementar reformas que no han sido suficientes para mejorar la capacidad penitenciaria, la rehabilitación y la reinserción de los internos.

En tercer lugar, la fuerza de cambio en los sistemas adaptativos complejos implica el "flujo" de algún medio ${ }^{23} y$, de manera similar al Derecho

16. Martin Lodge y Kai Wegrich, "Introduction: Governance Innovation, Administrative Capacities, and Policy Instruments," en The Problem-Solving Capacity of the Modern State Governance Challenges and Administrative Capacities, eds. Martin Lodge y Kai Wegrich (Oxford: Oxford University Press, 2014), 11-14.

17. Thomas E. Webb, "Tracing an Outline of Legal Complexity", Ratio Juris 27, no. 4 (2014); Donald T. Hornstein, "Complexity Theory, Adaptation, and Administrative Law», Duke Law Journal 54, no. 4 (2005). Nuestra investigación considera el enfoque de Webb (2014) que hace una distinción entre la Teoría de la Complejidad, la Teoría del Caos y la Teoría de la Autopoiesis. Sin embargo, otros académicos citados por Webb como J.B. Ruhl, J. Salzman, P. Cilliers, J. Murray, Julian Webb, N. Leuhmann y G. Teubner, entre otros, realizaron investigaciones sobre la Teoría de la Complejidad, políticas públicas y el Derecho. La Teoría de la Complejidad es empleada para examinar el Derecho Administrativo por académicos como Ruhl y Salzman (Hornstein, 2005, 914), así como Hornstein (2005). Además, otros autores exploran la Teoría de la Complejidad en diferentes áreas del derecho: Ruhl examina la Teoría de la Complejidad en la normativa medioambiental, sus fallos, y los mecanismos para mejorar su eficacia (Webb, 2014, 482), Roe analiza dicha teoría en relación con el derecho corporativo, y Di Lorenzo explora la Teoría de la Complejidad a través de la teoría del caos para mejorar la legislación (Hornstein, 2005, 914).

18. Aaron Pycroft y Clemens Bartollas, "Introduction" en Applying Complexity Theory: Whole Systems Approaches to Criminal Justice and Social Work, eds. Aaron Pycroft y Clemens Bartollas, (Bristol: Policy Press, 2014$) 2$.

19. J.B. Ruhl, "Thinking of Environmental Law as a Complex Adaptive System: How to Clean Up the Environment by Making a Mess of Environmental Law", Houston Law Review 34, no. 4 (1997).

20. John Henry Holland, Hidden Order: How Adaptation Builds Complexity (Nueva York: Perseus Books, 1995).

21. Ruhl, Thinking of Environmental Law as a Complex Adaptive System, 942.

22. Ibíd, 946 .

23. Ibíd, 947. 
Ambiental, la información disponible del INPE así como las percepciones de la sociedad son consideradas por el Poder Ejecutivo y el Congreso para la elaboración de políticas y leyes en materia penitenciaria.

En cuarto lugar, la "diversidad" es generada por la perturbación en los sistemas adaptativos complejos $^{24}$ y está representada por los cambios en las políticas y reglamentos penitenciarios, así como por la decisión política del MINJUS y del INPE como líderes del sector.

Por último, la "autocrítica" se orienta a adaptar el cambio en una norma estable en lugar de una reforma radical a pesar de que el sistema podría experimentar ocasionalmente interrupciones importantes inherentes a la no linealidad y la agregación. ${ }^{25}$ Este caso es evidente en la declaración de emergencia de la prisión en los últimos años que se ha convertido en una situación normalizada en el Sistema Penitenciario.

Si bien los eventuales motines o disturbios durante alguna emergencia pueden ser controlados mediante diversos métodos de disuasión tales como la supervisión por el personal penitenciario, implementación tecnológica, medidas de seguridad física - torres y cercos-, informantes dentro de los internos e información de inteligencia, ellos no resultan suficientes en la práctica. ${ }^{26}$ Por tanto, si durante una epidemia, las medidas que suelen ser adoptadas para el confinamiento no deben comprender la suspensión de derechos humanos para aquellas personas en libertad, ${ }^{27}$ este criterio debe ser considerado de la misma manera para aquellas personas recluidas en establecimientos penitenciarios.

La prevención puede ser más conveniente y en algunos casos, mucho más crítica que el propio tratamiento de la misma como en caso de una epidemia pero dicha prevención requiere ser estrictamente fiscalizada para evitar el riesgo de reforzar situaciones existentes de inequidad. ${ }^{28}$ En ese sentido, el rol de la Defensoría del Pueblo como institución independiente en materia de supervisión penitenciaria es indiscutible para identificar aquellas situaciones de afectación de derechos humanos y promover la implementación de mecanismos para que dichas situaciones no se repitan.

Por ello, el aporte de Webb basado en el trabajo de Cilliers conjuntamente con el de Lodge y Wegrich es empleado para explorar el Sistema Penitenciario bajo complejidad en cuanto a su capacidad normativa bajo los límites - boundaries-, capacidad analítica bajo el concepto de auto-organización -self-organization-, capacidad de entrega de resultados bajo los atractores - attractors-, y la capacidad de coordinación por su relación con el entorno y la adaptación del sistema ${ }^{29}$ en un contexto en el cual Sistema Penitenciario peruano es un sistema adaptativo complejo, sujeto a supervisión por la Defensoría del Pueblo.

\section{CÁRCELES PERUANAS EN CRISIS Y LA SU- PERVISIÓN PENITENCIARIA}

La finalidad del Sistema Penitenciario Peruano

24. Ibíd, 989.

25. Ibíd, 943, 952.

26. Richard H. Rison y Peter M. Wittenberg, "Disaster Theory: Avoiding Crisis in a Prison Environment", Federal Probation 58, no. 3 (1994): 45.

27. Chris Dibben, "Human epidemic", en The Routledge Handbook of Harzards and Disaster Risk, eds. Ben Wisner, JC Gaillard e llan Kelman (Abingdon: Routledge, 2012): 367.

28. Ibid, 371.

29. Webb, Tracing an Outline of Legal Complexity, 491. 
está asociada al tratamiento y reinserción del interno bajo custodia en adecuadas penitenciarias. Por otro lado, algunos autores consideran otros aspectos en relación con los asuntos penitenciarios y la justicia penal como el asistencialismo penal, ${ }^{30}$ la readaptación, la repersonalización, la rehabilitación sociocultural y la resocialización, ${ }^{31}$ la normalización de la vida en prisión, ${ }^{32}$ o la prevención y la seguridad. ${ }^{33}$ No obstante ello, los políticos son más directivos, los expertos penales menos influyentes, y la opinión pública se convierte en un punto de referencia clave en la evaluación para la toma de decisiones..$^{34}$ Por ello, las políticas y legislación penitenciaria peruana se encuentran sujetas a cambios o reversión debido al estado de ánimo de la opinión pública.

Ante esta situación, el rol de la Defensoría del Pueblo resulta fundamental en la supervisión del
Sistema Penitenciario. El impacto de las acciones de dicha institución en América Latina es "muy relativo", y el cumplimiento de las recomendaciones del Ombudsman por parte de los organismos estatales es limitado debido a su mandato no coercitivo. ${ }^{35}$ Por lo tanto, el desarrollo de las defensorías del pueblo está sujeto al contexto político en el que se desarrollan como institución y puede variar a lo largo de América Latina.

En el caso de la Defensoría del Pueblo peruana, esta fue creada a partir de la Constitución de 1993 con el mandato constitucional conferido por el Estado de proteger el derecho del ciudadano en todo el país: (i) defender los derechos constitucionales y fundamentales de la persona y de la comunidad, (ii) vigilar el cumplimiento de los deberes de función de la Administración Pública y (iii) controlar los servicios públicos prestados a la población. ${ }^{36}$ Por otro

30. De acuerdo con David Garland define el "asistencialismo penal" — penal welfarism — como los ideales de rehabilitación y la suposición de que la mejora del entorno económico y social para las personas de bajos ingresos reduce el comportamiento delictivo. Cfr. Carina Gallo y Mimi E. Kim, Crime Policy and Welfare Policy, en Oxford Handbooks Online (Nueva York: Oxford University Press, 2016), 2.

31. Según Juan Abraham Ramos Suyo, Derecho de Ejecución Penal y Administración Penitenciaria, cuarta edición (Lima: Editorial y Librería Jurídica Grijley E.I.R.L., 2016), 132-140, el interno será sometido a un proceso de readaptación - construcción de la conciencia, eliminación de los traumas mentales, deseos y frustraciones asociados a la conducta delictiva-, de repersonalización —recuperación de la personalidad individual del interno y de todos sus derechos—, de rehabilitación sociocultural —incluyendo la capacidad jurídica_ - y de resocialización — reinserción en la sociedad con pleno respeto a la vida, la salud y los bienes-.

32. José Cid y Ariadna Andreu, "European Prison Policy and Spanish Prison Practices: Understanding Confluences and Gaps", en Europe in Prisons: Assessing the Impact of European Institutions on National Prison Systems, eds. Tom Daems y Luc Robert (Cham: Palgrave Macmillan, 2017): 264-271, señala que la normalización se refiere a que los derechos de los internos y las condiciones de vida en prisión no deben diferenciarse de las personas que viven sin mandato de internamiento y considera aspectos como el alojamiento, la atención sanitaria y el trato respetuoso ; Según Michael Adler y Brian Longhurst, Discourse, power and justice: Towards a new sociology of imprisonment (Londres: Routledge, 1994), 39, debido a las críticas al paradigma de la "rehabilitación", el enfoque de la "normalización" se basa en asegurar que el recluso sea considerado como una persona normal que ha cometido un delito y que debe ser castigado por ese comportamiento delictivo. La vida en prisión no le convertirá en una persona peor, pero sí significa necesariamente que durante el internamiento el recluso puede ser mejor.

33. Según David Garland, The culture of control: crime and social order in contemporary society (Chicago: The University of Chicago Press, 2001), 171, la prevención y la seguridad se basan en la reducción del daño y la gestión del riesgo para prevenir los factores que generan los delitos y tienen como objetivo reducir las posibilidades de cometer delitos, mejorar los controles y excluir el comportamiento de las situaciones delictivas en lugar de promover la retribución, la disuasión y la reforma de los reclusos.

34. Ibíd, 172.

35. Uggla, The Ombudsman in Latin America, 449

36. Constitución Política del Perú, artículo 162. 
lado, la Ley Orgánica del Defensor del Pueblo considera que el Defensor del Pueblo deberá (iv) promover los Derechos Humanos, e (v) implementar y ejecutar el MNP en el marco del PFCCT. Sin perjuicio de ello, la promoción de los derechos humanos debe entenderse como un rol permanente para preservar los valores éticos en la administración pública, la convivencia social, así como la democracia institucional. ${ }^{37}$

La Defensoría del Pueblo ha promovido un enfoque de Derechos Humanos en democracias frágiles, y en el contexto peruano, dicho rol ha sido reconocido durante el gobierno de transición tras el tercer gobierno de Fujimori. Si bien no puede ser considerado como el salvador de todos los problemas del Estado, ${ }^{38}$ los aportes de la Defensoría del Pueblo han resultado positivos en la sociedad peruana. ${ }^{39}$

Notablemente, el cumplimiento de las decisiones emitidas por la Defensoría del Pueblo representa un desafío dada su naturaleza no vinculante. Mientras que más del 80 por ciento de los casos se resuelven de acuerdo con las recomendaciones del Defensoría del Pueblo peruana, el porcentaje cambia drásticamente a menos del 10 por ciento en el caso de que dichas propuestas sugieran compensaciones monetarias. ${ }^{40}$
Actualmente, la Defensoría del Pueblo cuenta con dos áreas a relacionadas con los temas penitenciarios: el Programa de Asuntos Penales y Penitenciarios realiza un seguimiento integral de todos los asuntos relacionados con las prisiones, excluyendo las funciones relativas a la prevención de los malos tratos y situaciones posteriores a dichos eventos, los cuales se encuentran bajo la supervisión de la Dirección del MNP, de conformidad con el PFCCT.

Las obligaciones del PFCCT se enfocan en la afectación de los derechos humanos relativos a la prevención de los malos tratos durante la detención, ${ }^{41}$ pero excluye aquellas cuestiones del funcionamiento y mantenimiento de las prisiones..$^{42}$ Dentro de los aspectos relacionados con los Derechos Humanos, la implementación del MNP tiene como objetivo mejorar las medidas de prevención para garantizar los tratos adecuados hacia aquellas personas privadas de libertad ${ }^{43}$ y no necesariamente considera calificar las condiciones de alojamiento durante de privación de libertad, alimentación, salud, educación y tratamientos para la rehabilitación. En este contexto, cada Estado Parte del PFCCT permitirá al MNP visitar cualquier lugar bajo su jurisdicción en el que haya o pueda haber personas privadas de su libertad por cualquier forma de detención o encar-

37. Fernando Castañeda Portocarrero, "La Defensoría del Pueblo y su Contribución con la Democracia en el Perú", Derecho \& Sociedad 36 (2010): 294.

38. Uggla, The Ombudsman in Latin America, 449-450.

39. Uggla, The Ombudsman in Latin America, 423-450; Samuel Abad Yupanqui, "La Defensoría del Pueblo: La Experiencia Peruana", Teoría y Realidad Constitucional 26, (2010): 481-510; Castañeda, La Defensoría del Pueblo y su Contribución con la Democracia en el Perú, 293-298; Iván Lanegra Quispe, “La Defensoría del Pueblo del Perú y la calidad de la democracia", Politai 2, no. 2, (2011): 38-53

40. Uggla, The Ombudsman in Latin America, 441.

41. Protocolo Facultativo de la Convención contra la Tortura, y otros tratos o penas crueles, inhumanos o degradantes de la Organización de las Naciones Unidas, artículo 1.

42. Christine Bicknell y Malcolm Evans, "Monitoring Prisons: The Increasingly Complex Relationship Between International and Domestic Frameworks", en Europe in Prisons: Assessing the Impact of European Institutions on National Prison Systems, eds. Tom Daems y Luc Robert (Cham: Palgrave Macmillan, 2017$): 12$.

43. Ibíd. 
celamiento o la colocación de una persona en un entorno de custodia público o privado que no se le permita abandonar a voluntad por orden de cualquier autoridad judicial, administrativa o de otro tipo. ${ }^{44}$ La escasa investigación sobre los MNP ${ }^{45}$ provoca la necesidad de analizar el rol del Defensor del Pueblo como MNP y la supervisión de los establecimientos penitenciarios a cargo del INPE.

La implementación del MNP ha sido adecuada a las necesidades y la estructura política de cada país. En particular, el modelo "Ombudsman Plus" comprende la implementación del MNP mediante la designación de una institución nacional de derechos humanos ya existente como tal, a través de la asignación de dichas funciones a una división interna independiente, la creación de una unidad separada con funciones de MNP sujetas al control de gestión directa del Defensor del Pueblo, o bien, la segregación de las funciones del MNP a través del Defensor del Pueblo. ${ }^{46}$ Además, algunos MNP trabajan directamente con la sociedad civil o indirectamente a través de un panel asesor, desconcentran sus estructuras en el caso de los estados federales o a nivel regional, o implementan órganos separados que trabajan como MNP en el caso de estructuras políticamente descentralizadas. ${ }^{47}$

En este contexto, el modelo "Ombudsman Plus" ha sido considerado como una alternativa en la mayoría de los países europeos ${ }^{48}$ y también en América Latina. Perú, entre otros países latinoamericanos, ha seguido dicha tradición y la Defensoría del Pueblo ha recibido el mandato como MNP desde 2016, siendo la "Dirección del MNP" el área a cargo de asumir dichas funciones. Sin embargo, en comparación con otras experiencias como el caso del Reino Unido en el que su MNP está compuesto por 21 órganos estatutarios que incluyen a la sociedad civil entre otros, o en el caso de Dinamarca que realiza visitas bajo el MNP con la sociedad civil o de Hungría que cuenta con la participación de la sociedad civil en un panel asesor, la Defensoría del Pueblo peruana como MNP no genera un espacio que congregue a la academia, funcionarios públicos, políticos, Poder Judicial y Poder Ejecutivo para debatir y planificar sobre temas de justicia penal y penitenciaria de manera integral.

En relación la supervisión penitenciaria, la Defensoría del Pueblo elabora y presenta Informes Anuales de su gestión en general y del MNP, e informes especiales sobre prisiones. Sin embargo, las actuaciones relacionadas con el Programa de Asuntos Penales y Penitenciarios y el MNP se detallan brevemente en el Informe Anual del Defensor del Pueblo y hacen hincapié en las áreas que aún se mantienen como principal preocupación.

La Defensoría del Pueblo, como MNP, es un actor activo y relevante en los temas de supervisión penitenciaria nacional y dicha institución tiene un trabajo independiente y aislado para analizar e identificar los problemas y hacerlos visibles a la opinión pública. No obstante, los resultados de la Defensoría del Pueblo no han tenido un impacto eficiente en la autoridad en materia penitenciaria debido a la tardía o nula

44. Protocolo Facultativo de la Convención contra la Tortura, y otros tratos o penas crueles, inhumanos o degradantes de la Organización de las Naciones Unidas, artículo 4.

45. Conforme con Bicknell y Evans, "Monitoring Prisons: The Increasingly Complex Relationship Between International and Domestic Frameworks", la situación de los MNP es examinada en los países europeos.

46. Bicknell y Evans, 15 .

47. Ibíd.

48. Ibíd. 
respuesta del MINJUS o del INPE ${ }^{49}$ ante los requerimientos de información. Por lo tanto, el rol del Defensor del Pueblo en la práctica apunta a la emisión de informes y reclamos en los medios de comunicación pero sin una significativa influencia en el proceso de resolución de problemas dentro del Estado: es un observador crítico pero no se ha involucrado en una estrategia integral para crear conciencia y discusión entre los actores públicos y privados en materia penitenciaria como el MINJUS, el INPE y la sociedad civil. ${ }^{50}$

\section{SUPERVISIÓN Y REFORMAS PENITENCIA- RIAS DURANTE LA PANDEMIA}

La Defensoría del Pueblo ha permitido identificar los problemas del Sistema Penitenciario y ha emitido recomendaciones a las autoridades competentes desde su creación. Considerando que uno de mayores retos se refiere a la deficiente infraestructura penitenciaria, visibilizada reiteradamente en los Informe Anuales, Informe Especiales de Adjuntía y los Informes del MNP, es evidente que los gobiernos deberían considerar la asignación de recursos para el mejoramiento y construcción de nuevos establecimientos penitenciarios como prioridad. Sin embargo, ello no ha sucedido de la manera esperada ${ }^{51}$ y la llegada de la COVID-19 agravó las condiciones penitenciarias. Por ello, la Defensoría del Pueblo emitió tres informes especiales $^{52}$ sobre el sistema penitenciario en pandemia ante la insuficiente respuesta de las autoridades y resulta necesario analizar la capacidad normativa, analítica, de coordinación y entrega de resultados del Estado Peruano ante este contexto.

El Informe Especial N 03-2020-DP a cargo del Programa de Asuntos Penales y Penitenciarios indicó que, si bien la capacidad carcelaria es de 40,137 internos, las cárceles poseen una población de 97,111 , con un $140 \%$ de sobrepoblación carcelaria; y sugirió medidas en materia de salud, seguridad, tratamiento a personas privadas de libertad, reducción de la situación de hacinamiento, vigilancia y control y trabajadores penitenciarios. Sin embargo, debido a la escasa respuesta gubernamental, el Informe Especial $N^{\circ}$ 08-2020-DP, también emitido por el Programa de Asuntos Penales y Penitenciarios, propuso acciones para que el Poder Ejecutivo reduzca los niveles de hacinamiento por el brote de COVID-19, tales como el indulto presidencial y la reforma legislativa - aumentar el acceso a la conversión de penales, internos por omisión de alimentos, cambios en la prisión preventiva y vigilancia electrónica personal-, así como incentivar a la Fiscalía y al Poder Judicial para que establezcan criterios de evaluación de los procesos procesales y judiciales vinculados al uso racional y limitado de las detenciones preventivas considerando el contexto carcelario y de

49. Carlos Eduardo Fernández Millán —abogado y Jefe del Programa de Asuntos Penitenciarios de la Defensoría del Pueblo- en discusión con el autor, julio 2020; Federico Javier Llaque Moya —abogado y exvicepresidente del INPE-, en discusión con el autor, julio 2020; Víctor García Toma —abogado, expresidente del Tribunal Constitucional y exministro de Justicia- en discusión con el autor, julio 2020.

50. César Augusto Nakazaki Servigón —abogado y profesor universitario—en discusión con el autor, agosto 2020.

51. El Plan Nacional de Infraestructura, aprobado mediante Decreto Supremo $N^{\circ}$ 238-2091-EF, no contempla al sector penitenciario dentro de sus proyectos priorizados.

52. Defensoría del Pueblo: Situación de las Personas Privadas de Libertad a Propósito de la Declaratoria de Emergencia Sanitaria, Serie Informes Especiales 03-2020-DP, 6 de abril de 2020, https://www.defensoria.gob.pe/wp-content/uploads/2020/04/Serie-de-Informes-Especiales-N\%C2\%BA-003-2020-DP.pdf; Tema II: Medidas para reducir el hacinamiento en el sistema penitenciario frente a la emergencia generada por la COVID-19, Serie Informes Especiales 08-2020-DP, 21 de abril de 2020, https://www.defensoria.gob.pe/wp-content/uploads/2020/04/Serie-de-Informes-Especiales-N\%C2\%BA-008-2020-DP.pdf; Informe Especial 4: Condiciones de las personas privadas de libertad en el contexto de emergencia sanitaria por Covid-19, Junio 2020, https://www.defensoria.gob.pe/ wp-content/uploads/2020/08/INFORME-ESPECIAL-N\%C2\%BA-4.pdf 
acuerdo con los estándares fijados por la Corte Interamericana de Derechos Humanos, la Corte Constitucional y la Corte Suprema.

En cuanto a los informes del MNP, la Defensoría del Pueblo ha señalado la importancia de resolver los desafíos penitenciarios en cuanto a la sobrepoblación de las cárceles, el uso prolongado de la prisión preventiva y los derechos humanos de las internas, pues intentan preservar el buen trato hacia la población penitenciaria. ${ }^{53}$ Por ello, observamos que el MNP ha priorizado la vigilancia de los menores infractores y de las internas como objeto de supervisión permanente, encontrándose éstas últimas a cargo de la autoridad del INPE. ${ }^{54}$ No obstante, el Informe Especial $N^{\circ} 4$ del MNP fue emitido durante el brote de COVID-19 visibilizó que el problema de hacinamiento carcelario debe ser resuelto a la brevedad y que la atención médica, alimentación y provisión de agua representan una deficiencia mayor en las cárceles que provocó una reacción violenta en los internos para llamar la atención de las autoridades gubernamentales. Lamentablemente, este informe no incluyó mención alguna sobre las visitas realizadas a establecimientos penitenciarios y representa una gran ausencia por parte de la Defensoría del Pueblo.

A pesar de que el mandato del MNP no sólo comprende los asuntos penitenciarios, sino también cualquier otra situación en la que haya personas privadas de libertad y que dicho MNP pueda supervisar también estas otras áreas como los centros de detención de menores, los centros de detención temporal de la Policía y los centros de salud que puedan tener pacientes bajo su custodia, es claro observar que la Defensoría del Pueblo no necesariamente estaría supervisando adecuadamente la situación penitenciaria, hallazgos y análisis respecto a los problemas penitenciarios que son comúnmente revisados por el Programa de Asuntos Penales y Penitenciarios. No podemos negar que la Defensoría del Pueblo del Perú presenta mejoras en su rol de supervisión al ser designada como MNP, pero si dicho rol cubre la totalidad de los establecimientos penitenciarios, éstos no necesariamente reflejarían todos y cada uno de los problemas y el contexto actual penitenciario en detalle.

Las dificultades de la Defensoría del Pueblo, sin embargo, no son exclusivas de dicha institución sino por la capacidad del Estado Peruano. Por ello, es necesario analizar la capacidad normativa, analítica, de entrega de resultados y de coordinación.

\section{Capacidad normativa.}

Webb ${ }^{55}$ considera que los límites definen el Sistema, ya sean sistemas parciales o límites observados del sistema parcial, y los sistemas no adaptan necesariamente su estructura o límites hacia las externalidades a menos que se consideren "legales", aunque el concepto de "legal" puede cambiar de vez en cuando. Por lo tanto, los límites del derecho se refieren al "producto de la interacción emergente, del encuentro entre las autocomprensiones de los conjuntos, los pro-

53. Defensoría del Pueblo, Primer Informe Anual: Mecanismo Nacional de Prevención de la Tortura y otros Tratos o Penas Crueles, Inhumanos o Degradantes, mayo 2017, https://www.defensoria.gob.pe/wp-content/ uploads/2018/05/Informe_tortura_2016.1.pdf

54. Ibíd; Defensoría del Pueblo: Segundo Informe Anual: Mecanismo Nacional de Prevención de la Tortura y otros Tratos o Penas Crueles, Inhumanos o Degradantes, junio 2018, https://www.defensoria.gob.pe/wp-content/ uploads/2018/09/Informe_tortura_2018.pdf ; Tercer Informe Anual: Mecanismo Nacional de Prevención de la Tortura y otros Tratos o Penas Crueles, Inhumanos o Degradantes, mayo 2019, https://www.defensoria.gob.pe/ wp-content/uploads/2019/07/Tercer-InformeAnual-MNPT.pdf ; Cuarto Informe Anual: Mecanismo Nacional de Prevención de la Tortura y otros Tratos o Penas Crueles, Inhumanos o Degradantes, mayo 2020, https://www. defensoria.gob.pe/wp-content/uploads/2020/05/InformeAnual_MNPT2020_7.pdf

55. Thomas E. Webb, “Exploring System Boundaries", Law and Critique 24, no. 2, (2013): 488. 
cesos y los conceptos que reclaman la legitimidad otorgada por identificarse como legales, y aquellos conjuntos obligados a comprometerse con ellos; otros procesos sociales y personas". ${ }^{56} \mathrm{Sin}$ embargo, los límites influyen en la adaptación de las reglas y la estructura organizativa del sistema debido a su capacidad de aprendizaje y a la memoria de los participantes del sistema, tal y como propone Cilliers. ${ }^{57}$

En caso de que se excluyan los problemas derivados de contextos no lineales, el sistema jurídico puede verse influenciado debido a la capacidad de aprendizaje, en aspectos como una "memoria limitada", la "localidad y contingencia del conocimiento", y las imperfecciones innatas de sus modelos ${ }^{58}$ y por tanto, los resultados pueden ser imprevisibles.

En tal sentido, nuestra Constitución ${ }^{59}$ y el Código de Ejecución Penal ${ }^{60}$, establecen que el Sistema Penitenciario Peruano se centra en el tratamiento y reinserción del interno y en el estado óptimo de las instalaciones penitenciarias.

Sin perjuicio de ello, ante la ausencia de un pronunciamiento por parte del Estado en materia penitenciaria, ${ }^{61}$ la Defensoría del Pueblo publicó el 6 de abril de 2020 su primer informe exhortando al gobierno a tomar medidas en el Sistema Penitenciario en áreas tales como salud, seguridad, implementación de mecanis- mos para la comunicación de los internos y sus familiares, reducción de hacinamiento, monitoreo y control y sobre el personal penitenciario. ${ }^{62}$

Posteriormente, mediante Decreto Legislativo 1459, publicado el 14 de abril de 2020 se establece el mecanismo para optimizar la conversión de la pena para personas condenadas por el delito de omisión de asistencia familiar y a los pocos días, la Defensoría del Pueblo emite su segundo informe sobre la situación penitenciaria y la pandemia con fecha el 21 de abril de $2020,{ }^{63}$ planeando medidas para la reducción del hacinamiento por parte del Poder Ejecutivo - gracias presidenciales y reformas normativas tales como la ampliación de la conversión de la pena, ampliación de beneficios penitenciarios a aquellos sentenciados por omisión de asistencia familiar, cambios en la prisión preventiva y vigilancia electrónica familiar-y acciones a cargo del Ministerio Público y del Poder Judicial.

Luego de ello, el 23 de abril de 2020 se publica el Decreto Supremo No 004-2020-JUS que aprueba el procedimiento simplificado para la solicitud de gracias presidenciales y el 4 de junio de 2020 se publican los Decretos Legislativos 1513 y 1514 para la implementación de medidas para la reducción de hacinamiento y la optimización de la implementación de equipos de vigilancia electrónica. En dicho mes, a su vez, es publicado el Informe Especial del MNP

56. Thomas E. Webb, "Asylum and Complexity: the vulnerable identity of law as a Complex System", en Complexity Theory and Law: Mapping an Emergent Jurisprudence, eds. Jamie Murray, Thomas E. Webb y Steven Wheatley (Abingdon: Routledge, 2019) 72.

57. Webb, Exploring System Boundaries, 488.

58. Ibíd, 489.

59. Constitución Política del Perú, artículo 139, secciones 21-22.

60. Código de Ejecución Penal, Texto Preliminar, artículo III y artículo 3.

61. El Plan Nacional de Preparación y Respuesta al riesgo de la introducción del coronavirus aprobado mediante Resolución Ministerial 039-2020/MINSA no incluyo a la población privada de libertad.

62. Ver Defensoría del Pueblo, Informe Especial 03-2020-DP, Capítulo V: Conclusiones y Recomendaciones.

63. Ver Defensoría del Pueblo, Informe Especial 08-2020-DP, Capítulo VI: Propuestas de la Defensoría del Pueblo. 
sobre las acciones identificadas como malos tratos a la población peruana en el marco de la pandemia.

Por lo anteriormente expuesto, la Defensoría del Pueblo resalta que durante el brote de la COVID-19, sus recomendaciones fueron tomadas por el gobierno de turno para reducción de hacinamiento. ${ }^{64} \mathrm{Si}$ bien en anteriores casos resulta difícil determinar si las sugerencias de dicha institución fueron adoptadas por el Poder Ejecutivo, la pandemia ha permitido identificar esta relación causa-efecto, que si bien ha estado rodeado de críticas y de resultados insuficientes, representa un importante paso en la reforma penitenciaria y evidencia que, a pesar de la existencia de una fragilidad en el liderazgo político del Poder Ejecutivo y tensiones entre el gobierno y el Poder Legislativo, es posible la dación de leyes en favor del interés público.

\section{Capacidad analítica.}

Un sistema bajo complejidad se refiere a un sistema auto organizado en el que su propia organización surge repentinamente de un contexto no ordenado como resultado de diversas decisiones internas controladas por la interrelación de sus partes. Como consecuencia de las interrelaciones entre dichas partes y su entorno, el conjunto de la organización trasciende sus componentes y genera comportamientos auto organizados con un impacto no predecible como consecuencia de este contexto no lineal. ${ }^{65}$ Así, el INPE y el MINJUS son considerados dentro de la auto organización del Sistema Penitenciario debido a su capacidad legal de hacer cumplir las leyes y reglamentos del Poder Ejecutivo en materia de gestión penitenciaria, tratamiento de los internos y rehabilitación.

No obstante ello, INPE es un organismo ejecutor público dependiente del MINJUS responsable del Sistema Nacional Penitenciario ${ }^{66}$ que representa una carga para cualquier Ministro de Justicia en funciones: su continuidad, reputación y credibilidad están sujetas a cualquier posible crisis en las prisiones. ${ }^{67}$ En la práctica, el MINJUS opta por trasladar toda la responsabilidad y el riesgo al INPE en caso de que se produzca alguna crisis carcelaria ${ }^{68}$ y dicha situación fue evidente ante las grescas o agresiones, motines y reyertas ocurridas en el 2020 por el brote de COVID-19 en comparación con los años anteriores - ver Tabla 1-, sumada a la inestabilidad política del gobierno del presidente Martín Vizcarra, su vacancia, y los posteriores nombramientos de los presidentes Manuel Merino y Francisco Sagasti por parte del Poder Ejecutivo, y los continuos cambios del Presidente del INPE. ${ }^{69}$

64. Carlos Eduardo Fernández Millán, "Retos y desafíos del Sistema Penitenciario Durante y Después de la Pandemia", sábado 18 de julio de 2020, webinar, Defensoría del Pueblo, https://www.facebook.com/defensoriaperu/ videos/225372545184095/

65. Webb, Exploring System Boundaries, 485-487.

66. De conformidad con el Código de Ejecución Penal, artículo 133, la Ley de Organización y Funciones del Ministerio de Justicia, Ley 29809, artículo 15, y el Decreto Legislativo que fortalece el Sistema Penitenciario Nacional y el Instituto Nacional Penitenciario (INPE), Decreto Legislativo 1328, artículo 6.

67. José Luis Pérez Guadalupe - expresidente del INPE y exministro del Interior- en discusión con el autor, febrero 2020; García Toma, en discusión con el autor; Llaque Moya, en discusión con el autor; Nakazaki Servigón, en discusión con el autor.

68. García Toma, en discusión con el autor.

69. Durante los meses de marzo y diciembre 2020, INPE ha tenido 4 presidentes del Consejo Penitenciario: César Cárdenas, Gerson Villar, Ricardo Castillo y Susana Silva. 
Tabla 1. Gresca o Agresión, Reyerta y Motín en el periodo 2016-2020

\begin{tabular}{c|c|c|c|c} 
Año & $\begin{array}{c}\text { Gresca } \\
\text { o Agresión }\end{array}$ & Reyerta & Motín & Total \\
\hline 2016 & 117 & 8 & 0 & 125 \\
\hline 2017 & 175 & 5 & 3 & 183 \\
\hline 2018 & 175 & 6 & 4 & 185 \\
\hline 2019 & 261 & 8 & 1 & 270 \\
\hline 2020 & 238 & 16 & 18 & 272
\end{tabular}

Fuente: INPE, Dirección de Seguridad Penitenciaria

Ante la dificultad del INPE y el Ministerio de Justicia en evaluar las previsiones y acciones requeridas en materia penitenciaria durante la pandemia, la Defensoría del Pueblo manifestó que las autoridades gubernamentales no han tenido la capacidad técnica y política necesaria para reaccionar a tiempo contra la COVID-19,70 informó sobre la ocurrencia de malos tratos durante la pandemia a través de la Dirección del MNP, y propuso recomendaciones a las autoridades públicas en diversas materias, entre ellas los asuntos penitenciarios. ${ }^{71}$ La falta de transparencia del INPE durante la pandemia ha sido uno de los principales desafíos y tareas pendientes la institución.

\section{Capacidad de entrega de resultados.}

Los atractores refieren a donde se dirigen los resultados del sistema y contribuyen a la es- tructura del entorno complejo describiendo las "influencias sociales" y "estructuras" prevalecientes en una sociedad para construir una interpretación que permita entender las razones que generaron la ocurrencia de ciertos eventos en un momento específico. ${ }^{72}$ En particular, los atractores del Sistema Penitenciario pueden ser examinados bajo la Teoría de la Complejidad como parte de la capacidad administrativa en la entrega de resultados.

El régimen penitenciario tiene como objetivo la reeducación, rehabilitación y reinserción del interno en la sociedad al momento de su liberación. ${ }^{73}$ Reconoce que ningún interno será víctima de violencia moral, mental o física, ni será sometido a torturas o tratos inhumanos o degradantes. ${ }^{74}$ Sin embargo, los atractores del Sistema Penitenciario difieren del escenario ideal estipulado en las leyes y la Defensoría del Pueblo peruana es la institución pública independiente para monitorear dicho contexto.

Si bien el número de procesados recluidos en cárceles ha sido reducido de 35,892 personas —Diciembre 2012- a 34,879 —Diciembre 2019-, el incremento de sentenciados de 25,498 -Diciembre 2012-a 60,669 —Diciembre 2019 — ${ }^{75}$ representa una mejoría para la justicia penal dado que existe una mayor población penitenciaria con sentencia. Sin embargo, ello afecta significativamente a la capacidad de albergue y algunos académicos y profesionales consideran que este aspecto debe ser aborda-

70. Fernández Millán, en discusión con el autor.

71. Ver Defensoría del Pueblo, Informe 4, Capítulo VIII.

72. Webb, Exploring System Boundaries, 489.

73. Constitución Política del Perú, artículo 139, secciones 21-22.

74. Constitución Política del Perú, artículo 2, sección 24, literal h.

75. Instituto Nacional Penitenciario; Informe Estadístico Diciembre 2012 (2012), https://www.inpe.gob.pe/normatividad/estad\%C3\%ADstica/2012/369-diciembre2012/file.html ; Informe Estadístico Diciembre 2019 (2019) https:// www.inpe.gob.pe/normatividad/documentos/4295-informe-estadistico-diciembre-2019/file.html 
do a través de una reforma integral por parte del Poder Judicial y el Congreso. ${ }^{76}$

Al 15 de marzo de 2020, fecha en la que se anunció la declaración de emergencia, la tasa de hacinamiento en las cárceles era del $141 \%$, y el escenario ideal era reducir el exceso de 56,882 internos para controlar los efectos de la pandemia en todas las cárceles del país. Según la Unidad Estadística de INPE, en los meses de junio, julio y agosto se presentó un incremento de egresos de los establecimientos penitenciarios, siendo aplicada la remisión condicional de la pena conforme al Decreto Legislativo 1513 a partir del mes de julio ver Tabla 2-.

Tabla 2. Personas Privadas de Libertad que Egresaron entre marzo-noviembre 2020

\begin{tabular}{l|c|c|c|c|c|c|c|c|c}
$\begin{array}{l}\text { Población } \\
\text { Penitenciaria }\end{array}$ & Mar & Abr & May & Jun & Jul & Ago & Set & Oct & Nov \\
$\begin{array}{l}\text { Procesado } \\
\text { Sentenciado }\end{array}$ & 684 & 520 & 1,214 & 1,032 & 973 & 879 & 656 & 593 & 457 \\
\hline Total General & 1,057 & 1,281 & 2,573 & 2,343 & 2,326 & 2,300 & 1,940 & 1,675 & 1,388
\end{tabular}

Fuente: INPE, Unidad de Estadística

Las expectativas del gobierno y los resultados al promulgar las leyes durante la pandemia siguiendo las sugerencias de la Defensoría del Pueblo para la reducción del hacinamiento muestran que, si bien la formulación legislativa es posible de ser emitida durante periodos de inestabilidad política, existen serias dificultades en su implementación dado que involucra no sólo al INPE sino también al Ministerio de Justicia y al Poder Judicial. Por tanto, es necesario considerar que la supervisión penitenciaria no solo debería tomar en cuenta la elaboración y formulación normativa sino también los mecanismos para su implementación.

La percepción ciudadana es esencial para determinar el éxito o fracaso de políticas durante una emergencia y aspectos tales como la atención de los ciudadanos hacia los desastres, su entendimiento de la respuesta gubernamental y su comportamiento son necesarios de tomar en cuenta. ${ }^{77} \mathrm{Si}$ bien resulta prematuro determinar si las expectativas ciudadanas han sido satisfechas en materia penitenciaria, más aún tomando en cuenta que el resguardo de los derechos humanos de los internos puede generar opiniones contrapuestas, la percepción ciudadana es un aspecto que debe tomarse en cuenta en los resultados que se esperan alcanzar.

\section{Capacidad de coordinación.}

Los sistemas complejos pueden verse afectados debido a la interacción entre el entorno y las partes del sistema, y Webb considera que los trastornos del sistema pueden ser causados por la inadaptación y el estancamiento. La primera se refiere a que los sistemas no necesariamente se adaptarán adecuadamente debido a la respuesta a los factores ambientales sujetos al conocimiento de la población: las leyes mal adaptadas pueden parecer irrelevantes, dema-

76. Pérez Guadalupe, en discusión con el autor; García Toma, en discusión con el autor; Llaque Moya, en discusión con el autor; Nakazaki Servigón, en discusión con el autor.

77. Saundra K. Schneider, "Governmental Response to Disasters: Key Attributes, Expectations and Implications", en Handbook of Disaster Research, Segunda edición, eds. Havidán Rodríguez, William Donner y Joseph E. Trainor, (Cham: Springer, 2018) 560-562. 
siado rígidas, demasiado flexibles, inexactas o desarticuladas, para aquellos a quienes se aplica la regla. ${ }^{78}$ No obstante, esto último se refiere a que las reglas se olvidan, no se aplican mal ni se reconocen, sino que pasan desapercibidas. ${ }^{79}$ Por lo tanto, los participantes y su libre albedrío en un sistema complejo definen la dirección hacia la que se orienta dicho sistema, y dicha dirección puede quedar bloqueada en un camino de desarrollo particular, a pesar de su no linealidad, a menos que un "trastorno ambiental masivo obligue a un cambio significativo". ${ }^{80}$

En ese contexto, las relaciones entre el INPE y el Ministerio de Justicia con las cortes de justicia, el Congreso y la Defensoría del Pueblo presentan particularidades y han influido, directa o indirectamente, en el Sistema Penitenciario.

En primer lugar, consideramos importante explorar la influencia del Poder Judicial y el Tribunal Constitucional en el Sistema Penitenciario. La independencia y la discrecionalidad de los jueces para decidir si imponen o no prisiones preventivas, ${ }^{81}$ así como para determinar la duración de la pena una vez que una persona es declarada culpable, se reflejan en la población penitenciaria. Las personas detenidas durante la fase de investigación — previa a la acusación penal- o de enjuiciamiento, además de los sentenciados, afectan significativamente la capacidad de infraestructura y el proceso de reinserción dado que los programas de tratamiento enfocados en aspectos de educación y trabajo no resultan suficientes para atender a toda la población penitenciaria.

Ante dicha situación, la Corte Suprema de Justicia emitió el Acuerdo Plenario 01-2019/CIJ116 con fecha 10 de septiembre de 2019 para establecer un criterio estándar para las detenciones preventivas por parte de los jueces. Sin embargo, las prisiones preventivas aún son mantenidas en la práctica y si bien pueden ser revocadas, dicho proceso de revocación toma tiempo considerando la variable carga procesal de cada juzgado de turno.

En segundo lugar, el Congreso legisla mediante su única cámara en un Estado semi presidencialista bajo un sistema multipartidista desde la Constitución de 1993. Como consecuencia de las elecciones al Congreso y a la Presidencia de la República de 2016, el Poder Legislativo quedó atípicamente liderado por el único partido de la oposición, que obtuvo 73 de los 130 escaños. Dicho contexto generó tensiones entre el Congreso y el Poder Ejecutivo en el que el presidente Kuczynski renunció, el vicepresidente Vizcarra asumió la presidencia, el Congreso fue disuelto y los nuevos parlamentarios iniciaron funciones en marzo de 2020 en el contexto de la pandemia. Al inicio de la pandemia, el Poder Ejecutivo no tenía representación oficial en el Congreso ${ }^{82}$ y las tensiones entre ambos poderes del Estado eran una práctica habitual.

78. Webb, Tracing an Outline of Legal Complexity, 491.

79. Ibíd, 492.

80. Webb, 2014, 491.

81. Véase el Decreto Legislativo 957, Código Procesal Penal, artículo 268.

82. El vicepresidente Martín Vizcarra asumió la presidencia tras la renuncia de Pedro Pablo Kuzcynski el 23 de marzo de 2018 y disolvió el Congreso el 30 de septiembre de 2020 argumentando un voto de confianza negado por los congresistas. Sin embargo, el Tribunal Constitucional mediante sentencia del 14 de enero de 2020 (Expediente No 0006-2019-CC/TC) ratificó dicha decisión y los nuevos congresistas iniciaron funciones para el periodo comprendido entre el 19 de marzo de 2020 y el 26 de julio de 2021. Durante este periodo, los conflictos políticos entre el Poder Ejecutivo y el Congreso fueron frecuentes, el partido oficialista —Peruanos por el Kambio- rompió relaciones con el presidente Vizcarra y el Poder Ejecutivo no tuvo congresistas en el nuevo parlamento para el periodo 2020-2021. 
La primera iniciativa entre el Congreso y el Poder Ejecutivo fue la delegación de ciertas facultades en temas como salud pública, política fiscal y tributaria, promoción de inversiones, seguridad ciudadana y orden interno, protección de los derechos laborales, educación, prevención y protección de las personas en situación de vulnerabilidad, provisión de bienes y servicios a la población protección de las industrias, cultura y turismo por 45 días calendario mediante la Ley 31011 publicada el 27 de marzo de 2020. Posteriormente, se emitieron los Decretos Legislativos $1459^{83}, 1513^{84}$ y $1514^{85}$.

En tercer lugar, la Defensoría del Pueblo ha participado activamente en los desafíos que enfrentaba la población en materia de salud y seguridad debido a las restricciones gubernamentales por la pandemia. En el marco de esta participación, la institución emitió informes sobre la situación carcelaria, entre otros, y sugirió que el tema de la sobrepoblación se abordara de manera prioritaria, y que el Gobierno implementara acciones en materia de salud y reducción del entorno de sobrepoblación ante fácil contagio de la COVID-19. Debido a la proximidad del informe de la Defensoría del Pueblo peruana y la respuesta gubernamental, consideramos que el contexto de la COVID-19 nos permite identificar la relevancia de dicha institución respecto a la realidad carcelaria y las acciones adoptadas por el Poder Ejecutivo.
Los fundamentos de las sugerencias la Defensoría del Pueblo en materia penitenciaria durante la pandemia se centran en una cooperación interinstitucional para abordar la problemática de manera integral en el conjunto del Estado, involucrando a la Policía, el Ministerio de Trabajo y el Ministerio de Relaciones Exteriores, el Poder Judicial, Fiscalía y MINJUS como principales actores del sistema de justicia penal, Ministerio de la Mujer y Población Vulnerable, Ministerio de Educación, Ministerio de Desarrollo e Inclusión Social, Ministerio de Salud, Ministerio de Cultura y Ministerio de Economía y Finanzas.

Dichas recomendaciones consideran la reforma integral del Sistema Penitenciario y, por ende, modificaciones a la normativa vigente de Ejecución Penal, incremento del presupuesto nacional en materia penitenciaria para infraestructura, seguridad, tratamiento - asistencia psicológica, educativa y sanitaria- y apoyo al cuidado de los hijos de las internas, y exhortar a los jueces a considerar las condiciones de salud mental del interno en su juzgamiento y sanción.

Sin embargo, es necesario resaltar que los informes de la Defensoría del Pueblo presentan limitaciones. Según el Informe Especial de la Defensoría del Pueblo del Perú, sólo 34 de 66 cárceles fueron visitadas en 2011, lo que representa el $80,06 \%$ de la población penitenciaria ${ }^{86}$ y sólo 54 de 69, en 2017, lo que representa el $92,52 \%$ de la población penitenciaria. ${ }^{87}$ Por lo

83. El Decreto Legislativo 1459 optimiza la aplicación de la conversión automática de la pena para personas condenadas por el delito de omisión de asistencia familiar, a fin de reducir el hacinamiento penitenciario y evitar contagios de COVID-19, publicado en el Diario Oficial El Peruano el 14 de abril de 2020.

84. El Decreto Legislativo 1513 implementa medidas excepcionales contra el hacinamiento penitenciario, publicado en el Diario Oficial El Peruano el 4 de junio de 2020.

85. El Decreto Legislativo 1514 optimiza la implementación de la vigilancia electrónica personal como mecanismo coercitivo y sanción penal para reducir el hacinamiento penitenciario, publicado en el Diario Oficial El Peruano el 4 de junio de 2020.

86. Defensoría del Pueblo, El Sistema Penitenciario: Componente clave de la seguridad y la política criminal. Problemas, retos y perspectivas, Informe Defensorial 154-2011/DP, octubre 2011, https://www.defensoria.gob.pe/ wp-content/uploads/2018/05/informe-154-FINAL.pdf

87. Defensoría del Pueblo, Retos del Sistema Penitenciario Peruano: Un diagnóstico de la realidad carcelaria de 
tanto, si bien la mayoría de la población penitenciaria suele ser cubierta bajo la supervisión de la Defensoría del Pueblo peruana, existen algunos internos excluidos y es necesario examinar si dichos informes deberían cumplir con una muestra estadística o cubrir a toda la población penitenciaria.

Por último, el entorno sociocultural representa un reto para el Sistema Penitenciario en cuanto a su legitimidad y a las percepciones de la población. Aunque no existe un consenso para definir el concepto de legitimidad organizacional, los conceptos comunes confluyen hacia una valoración sobre el comportamiento de una organización determinada por el receptor de dicho comportamiento y sujeta a reglas y normas del entorno cultural del receptor. ${ }^{88} \mathrm{En}$ particular, Suchman examina tres tipologías: la legitimidad pragmática — basada en la determinación de lo que una organización está entregando a la sociedad-, la legitimidad cognitiva - las organizaciones son aceptadas ya que no hay otra opción - y la legitimidad moral. ${ }^{89}$ Debido a la complejidad en los establecimientos penitenciarios, al no existir otro actor que no sea el INPE encargado de conducir los asuntos penitenciarios, la legitimidad del INPE y sus acciones vinculadas al Sistema Penitenciario se apoya en el enfoque cognitivo.

En ese contexto, podemos observar que no ha existido una coordinación adecuada entre los actores del Sistema Penitenciario previamente y durante la pandemia, dejando de lado aspectos claves para el manejo de emergencias tales como contar con la preparación y experiencia de profesionales ante emergencias, asignación de recursos, canales de comunicación y coordi- nación, protocolos para la toma de decisiones y procesos y liderazgo..$^{90}$ Por ello, resulta necesario evidenciar que la Defensoría del Pueblo y su supervisión han sido insuficientes.

\section{CONCLUSIONES}

La capacidad administrativa del Estado Peruano ha representado desafíos a lo largo de estos 200 años de vida republicana y el Sistema Penitenciario, entre otros sectores, es una tarea pendiente de solucionar. La Teoría de la Complejidad permite analizar al Sistema Penitenciario como un sistema adaptativo complejo y su supervisión doméstica a cargo de la Defensoría del Pueblo.

La capacidad del Estado peruano analizada en el Sistema Penitenciario, en su calidad de sistema adaptativo complejo, evidencia que la capacidad normativa ha sido ejercida en la formulación y dación legislativa a pesar de las reiteradas crisis e inestabilidad política. Sin embargo, la capacidad analítica, de entrega de resultados y de coordinación son limitadas y requieren un entendimiento del funcionamiento orgánico del Estado Peruano para que puedan cumplir con las expectativas ciudadanas.

La Defensoría del Pueblo durante la pandemia ha demostrado que puede liderar la promoción y protección de derechos humanos de todos los ciudadanos, y si bien sus recomendaciones poseen un carácter no vinculante, es un paso importante en la reforma del Sistema Penitenciario que las opiniones de dicha institución fueron recogidas por el Poder Ejecutivo en la modificación y promulgación de nueva normatividad para abordar el deshacinamiento de los

mujeres y varones, Informe de Adjuntía 006-2018-DP-ADHDP, diciembre 2018, https://www.defensoria.gob.pe/ wp-content/uploads/2019/04/Retos-del-sistema-penitenciario.pdf.

88. María José Canel y Vilma Luoma-aho, Public Sector Communication: Closing Gaps Between Citizens and Public Organizations (Hoboken: John Wiley \& Sons Inc., 2019) 139.

89. Canel y Luoma-aho, 142.

90. Schneider, Governmental Response to Disasters: Key Attributes, Expectations and Implications, 558-560 
establecimientos penitenciarios como una medida a corto plazo.

Sin perjuicio de ello, la Defensoría del Pueblo a través del Programa de Asuntos Penales y Penitenciarios y la Dirección del MNP presenta limitaciones para congregar a los actores del Estado Peruano y la sociedad civil para replantear las acciones y prioridades en materia penitenciaria. Siendo considerada la Defensoría del Pueblo como una reserva moral, el carácter no vinculante de sus recomendaciones la posiciona con un grado importante de legitimidad por parte de la ciudadanía y es necesario evaluar si la estructura del MNP bajo el Ombudsman Plus es el más adecuado para establecer reformas a largo plazo que permitan alcanzar los objetivos del Sistema Penitenciario.

Por tanto, consideramos las propuestas de reforma del Sistema Penitenciario deben también considerar la manera en cómo el MNP podría funcionar y generar un espacio de discusión entre el sector público y privado para la mejora de aquellas personas albergadas en establecimientos penitenciarios. 